\title{
CARACTERIZAÇÃO E USO DE MADEIRAS DE GALHOS DE ÁRVORES PROVENIENTES DA ARBORIZAÇÃO DE BRASÍLIA, DF
}

\section{WOOD BRANCHES CHARACTERIZATION AND USE OF THE LITY OF BRAZILIA URBAN TREES}

\author{
Ailton Teixeira do Vale ${ }^{1}$ Thaise Rachel Sarmento ${ }^{2}$ Alexandre Nascimento Almeida ${ }^{3}$
}

\section{RESUMO}

Este trabalho objetivou a caracterização da madeira de galhos provenientes de poda de árvores da arborização de Brasília e constou da investigação da possibilidade de seu uso como lenha e carvão vegetal. Foram estudadas 12 espécies de maior presença na arborização de Brasília. As espécies Persea americana, Spathodea campanulata, Ficus sp, Chorisia speciosa e Ochroma pyramidale foram caracterizadas como madeiras moles, com massa específica abaixo de $0,44 \mathrm{~g} / \mathrm{cm}^{3}$. Caesalpinea leiostachya, com $0,73 \mathrm{~g} / \mathrm{cm}^{3}$ caracterizou-se como madeira dura e Pterogyne nitens, Peltophorum dubium, Mangifera indica, Syzygium jambolana, Ligustrum lucidum e Paquira aquática, com valores entre 0,5 e $0,7 \mathrm{~g} / \mathrm{cm}^{3}$ estão no grupo das madeiras moderadamente duras. As espécies: Caesalpinea leiostachya, Syzygium jambolana, Ligustrum lucidum e Peltophorum dubium apresentaram os maiores valores para contração volumétrica, entre 13 e $15,5 \%$, enquanto os menores valores foram encontrados para Ficus $s p$. e $M$. indica com, respectivamente, 6,70 e $7,41 \%$. Uma característica favorável para o uso da madeira para produção energética é a maior quantidade de massa por unidade de volume, dessa forma, as espécies de maiores massas específicas seriam as mais indicadas. As demais espécies poderiam ser transformadas em briquetes, aumentando assim a quantidade de calor por unidade volumétrica. Outros usos seriam como: composto orgânico (compostagem) e para a confecção de pequenos objetos (cinzeiros, cabos de ferramentas, enfeites, porta-jóias).

Palavras-chave: resíduo; poda; madeira; arborização.

\begin{abstract}
This work had as its principal objective the characterization of branches coming from tree pruning for use as fire wood and charcoal. There were studied twelve species of the lity of Brazilia urban trees. The specie Persea americana, Spathodea campanulata, Ficus sp, Chorisia speciosa and Ochoma pyramidale with density below $0.44 \mathrm{~g} / \mathrm{cm}^{3}$, were classified as soft wood. Caesalpinea leiostachya, with $0.73 \mathrm{~g} / \mathrm{cm}^{3}$ was classified as hard wood and Pterogyne nitens, Peltophorum dubium, Mangifera indica, Syzygium jambolana, Ligustrum lucidum and Paquira aquática, with specific gravity between $0.5 \mathrm{e} 0.7 \mathrm{~g} / \mathrm{cm}^{3}$ were classified as intermediate hardness. Four species (C. leiostachya, S. jambolana, L.lucidum e P. dubium) showed larger contraction (between 13 and 15.5\%) than Ficus sp. and M. indica with, respectively, 6.70\% and 7.41\%. The species with larger specific gravity are preferred for energy yield. On the other hand, species with lowersmall specific gravity, can be utilized for briquet, organic compositing and small wood objects.
\end{abstract}

Key words: wave; poda; wood; arborization.

\section{INTRODUÇÃO}

O plantio de árvore em zona urbana tem outros objetivos além da ornamentação, e como segmento da silvicultura tem oferecido opções interessantes para as paisagens das cidades, atraindo pássaros e, com isso, diminuindo a incidência de insetos, melhorando a oxigenação do ar, evitando a estagnação das camadas baixas da atmosfera e melhorando assim o equilíbrio térmico. Dentro desse conceito, a árvore, segundo Jacinto (2001), é parte de um ecossistema florestal urbano.

A manutenção desse ecossistema é feita mediante tratos culturais, e a poda é uma das operações utilizadas, consistindo na supressão de uma parte da copa ou do sistema radicular da planta. No setor urbano

1. Engenheiro Florestal. Dr., Professor do Departamento de Engenharia Florestal, Faculdade de Tecnologia, Universidade de Brasília, Caixa Postal 04357, CEP 70910-900, Brasília (DF). ailtontv@unb.br

2. Engenheira Florestal, CLN 208, Bloco "C", Kit 220, Asa Norte, CEP 70850-530 Brasília (DF). taizsarmento@bol.com.br

3. Engenheiro Florestal., SHCGN 706N, Casa 39, Asa Norte, CEP 70770-747, Brasília (DF). alexfloresta@pop.com.br

Recebido para publicação em 2/09/2003 e aceito em 20/12/2005. 
de Brasília, diariamente são feitos cortes de galhos e árvores, chegando a seis caminhões/dia. Após uma seleção, o material fino é descartado e aquele de maior diâmetro é armazenado, sendo periodicamente moído e utilizado para coroamento de covas. A pouca utilização desse material talvez esteja na falta de conhecimento das opções de uso.

A opção de uso do resíduo florestal passa pelo conhecimento tecnológico da madeira, partindo de suas características físicas, químicas e mecânicas. Alta massa específica está associada à elevada dureza, indicando maior quantidade de substância madeira por unidade volumétrica, o que é vantajoso na produção de energia, tanto na forma de carvão vegetal quanto de lenha. Para peças maiores, o conhecimento da massa específica pode levar à indicação para confecção de pequenas estruturas de madeira. Os valores de contração podem indicar o uso para móveis e pequenos objetos. A análise química imediata pode ser utilizada para classificar as espécies para uso energético.

A variação da massa específica da madeira ocorre em conseqüência das variações na espessura da parede celular, ao tamanho das células, à inter-relação entre esses fatores e à quantidade de substâncias extratáveis presentes (Vital, 1984, citando Brown, 1952; Haygreen, 1982 e Panshin, 1982). Dessa forma, via de regra, madeiras com massas específicas maiores possuem paredes celulares mais espessas e lumens menores, portanto, poucos espaços vazios o que implica em menores umidades máximas. Madeiras com tal característica, ou seja, com maior massa específica, apresentam maiores resistências mecânicas, podendo ser utilizadas em estruturas, como vigas, colunas, móveis; e, quando utilizadas para a produção direta de calor, pela da combustão, liberam maior quantidade de calor por unidade volumétrica; quando utilizadas de forma indireta para a produção de calor, pela carbonização, produzem carvão de maior massa específica e, portanto maior concentração de calor por unidade de volume.

A massa específica, o teor de carbono fixo, o teor de umidade, a análise elementar e a análise imediata são indicados por Junge (1975), Arola (1976) e Corder (1976), citados por Brito \& Barrichelo (1978), como as propriedades mais importantes da madeira para sua utilização como combustível.

Diante do exposto, o trabalho teve por objetivo principal a caracterização da madeira de galhos provenientes da poda de árvores da arborização do Distrito Federal, mais especificamente, do Plano-Piloto, e a indicação de possíveis fins estes e como objetivos secundários, probabilidade de utilização desses galhos para produção de lenha e carvão vegetal e ainda a relação da massa específica e do rendimento gravimétrico com parâmetros de produção e qualidade do carvão vegetal daí resultante.

\section{MATERIAL E MÉTODOS}

\section{Escolha das espécies}

De acordo com o levantamento preliminar das espécies e do número de árvores, que ocorrem no Distrito Federal, realizado pela NOVACAP, para as Quadras de número 100 da Asa Sul, tem-se um total de 143 espécies. Baseado nesses dados, foram escolhidas, para este trabalho, 12 espécies, em função da maior ocorrência em número de árvores e, portanto, maior quantidade de material podado, conforme Tabela 1. 
TABELA 1: Espécies da arborização de Brasília utilizadas no estudo.

TABLE 1: Species from Brasília urban trees utilized in the study.

\begin{tabular}{lll|c}
\hline Nome Vulgar & \multicolumn{1}{c|}{ Nome Científico } & \multicolumn{1}{c}{ Família } & N. árv. \\
\hline 1. Abacateiro & Persea americana Mill. & Lauraceae & 4 \\
2. Amendoim-bravo & Pterogyne nitens Tul. & Leguminosa-Caesalpinoideae & 4 \\
3. Cambuí & Peltophorum dubium Taub. & Leguminosa-Caesalpinoideae & 2 \\
4. Espatódea & Spathodea campanulata Benv. & Bignoniacea & 3 \\
5. Fícus benjamina & Ficus sp. & Moraceae & 4 \\
6. Mangueira & Mangifera indica L. & Anacardiaceae & 3 \\
7. Pau-ferro & Caesalpinea ferrea Mart. ex Tul. var. & Leguminosa-Caesalpinoideae & 4 \\
8. Jambolão & leiostachya Benth. & Myrtaceae & 3 \\
9. Ligustro & Syzyium jambolana D.C. & Oleaceae & 3 \\
10. Monguba & Pachira aquatica Aubl. & Bombacaceae & 5 \\
11. Paineira & Chorisia speciosa ST. Hil. & Bombacaceae & 2 \\
12. Pau-de-balsa & Ochroma pyramidale (Cav. ex Lamb.) & Bombacaceae & 3 \\
\hline
\end{tabular}

\section{Amostragem}

Para cada espécie, foram amostradas, ao acaso, galhos provenientes de um mínimo de duas árvores. A amostragem foi feita de acordo com o trabalho diário executado pelos funcionários da Nova Capital (NOVACAP), empresa encarregada pela arborização de Brasília. De cada árvore, foram escolhidos três galhos com diâmetro superior a $5,0 \mathrm{~cm}$ na base, de tal forma que fossem contemplados, para cada árvore podada, galhos com maior diâmetro, com diâmetro intermediário e com menor diâmetro. De cada galho, a partindo de $50 \mathrm{~cm}$ de sua inserção no tronco, foram retirados dois toretes com comprimento de $20 \mathrm{~cm}$ cada ou um (1) torete de $40 \mathrm{~cm}$..

Esses toretes foram identificados e imediatamente colocados em embalagens plásticas sendo conduzidos, ao final de cada dia, para o laboratório onde foram armazenados, imersos em água.

Em seguida, os toretes foram cortados em discos, sendo que, para cada um dos discos de cada galho, foram retiradas duas cunhas opostas entre si procurando conter as zonas diametrais. Essas cunhas foram utilizadas para a determinação das massas específicas e contração volumétrica. Dessa forma, para cada galho, foram retiradas duas amostras, perfazendo um total de seis amostras por árvore e 18 por espécie. O restante do material foi colocado para secar ao ar livre para posterior carbonização e uso para análise imediata.

\section{Caracterização}

As amostras foram preparadas no Laboratório de Propriedades Físicas da Madeira da UnB e no Laboratório de Produtos Florestais (LPF) do IBAMA. Após o preparo (picagem, moagem e classificação em peneiras de 40 e 60 mesch para ensaio de análise imediata, segundo ASTM D-1762-64), realizou-se a caracterização da madeira.

A determinação das massas específicas foi feita segundo as metodologias propostas por Foelkel et al. (1971) e Vital (1984). A determinação da contração volumétrica máxima baseou-se na norma ABNT MB26/40. O teor de umidade foi determinado segundo metodologia proposta por Vital (1997).

Do material picado, uma parte foi destinada à carbonização e outra parte da madeira picada foi moída e homogeneizada em uma amostra composta por árvore. A fração de madeira moída foi classificada em peneira de 40 e 60 mesch. A análise foi feita em duplicata para cada espécie. Pela análise imediata, foi possível determinar os teores de material volátil (MV), de cinzas $(\mathrm{CZ})$ e, por diferença de carbono fixo $(\mathrm{CF})$, baseado na norma ASTM D-1762-64. 


\section{Carbonização}

Os discos foram picados e transformados em cavacos para que fosse garantida a maior uniformidade no tamanho das amostras para as carbonizações. Após secagem ao ar, os cavacos foram homogeneizados obtendo-se três amostras por espécie ou uma por árvore para espécies com duas árvores. As carbonizações foram conduzidas em laboratório, num tempo de 210 minutos, utilizando forno mufla à $450^{\circ} \mathrm{C}$, acoplado a um sistema de condensação para a recuperação do licor pirolenhoso, conforme Vale et al. (1996). O carvão e o licor pirolenhoso foram pesados, calculando-se, assim, o rendimento gravimétrico em carvão (RG), em licor pirolenhoso (RLP) e, por diferença, em gases não-condensáveis (RGNC).

\section{Análises dos dados}

Os dados obtidos nos testes realizados foram analisados estatisticamente mediante análise de variância e de regressão.

\section{RESULTADOS E DISCUSSÃO}

\section{Características da madeira}

A Tabela 2 apresenta os valores médios para massas específicas, umidades máximas e contrações das madeiras de galhos de podas de árvores da arborização de Brasília.

TABELA 2: Características das madeiras de galhos da poda de árvores da arborização de Brasília com seus desvios-padrão.

TABLE 2: Characteristics of wood branches from the city of Brasília pruned trees.

\begin{tabular}{|c|c|c|c|c|c|c|c|c|c|c|}
\hline \multirow[b]{2}{*}{ Amostra } & \multicolumn{6}{|c|}{ Massa específica da madeira $\left(\mathrm{g} / \mathrm{cm}^{3}\right)$} & \multirow{2}{*}{\multicolumn{2}{|c|}{\begin{tabular}{|c|} 
Umidade \\
máxima, base \\
seca $(\%)$
\end{tabular}}} & \multirow{2}{*}{\multicolumn{2}{|c|}{$\begin{array}{c}\text { Contração } \\
\text { volumétrica } \\
\text { máxima (\%) }\end{array}$}} \\
\hline & \multicolumn{2}{|l|}{ Saturada } & \multicolumn{2}{|c|}{ Básica } & \multicolumn{2}{|c|}{$\begin{array}{l}\text { A } 0 \% \text { de } \\
\text { umidade }\end{array}$} & & & & \\
\hline \multirow{2}{*}{ Persea americana } & 1,06 & \multicolumn{3}{|c|}{0,44} & \multicolumn{2}{|c|}{0,49} & \multicolumn{2}{|l|}{145,23} & \multicolumn{2}{|c|}{10,55} \\
\hline & 0,0589 & 12 & 0,0716 & 12 & 0,0838 & 12 & 33,5627 & 12 & 1,7260 & 12 \\
\hline \multirow{2}{*}{ Pterogyne nitens } & 1,09 & \multicolumn{3}{|c|}{0,59} & \multicolumn{2}{|c|}{0,66} & 83,91 & & \multicolumn{2}{|c|}{9,31} \\
\hline & 0,0604 & 12 & 0,0422 & 12 & 0,0472 & 12 & 13,6255 & 12 & 0,5642 & 12 \\
\hline \multirow{2}{*}{ Peltophorum dubium } & 1,18 & & \multicolumn{2}{|c|}{0,61} & \multicolumn{2}{|c|}{0,70} & 94,22 & & \multicolumn{2}{|c|}{13,26} \\
\hline & 0,0654 & & 0,0449 & 6 & 0,0799 & 6 & 9,7706 & 6 & 3,7155 & 6 \\
\hline \multirow{2}{*}{ Spathodea campanulata } & 0,99 & & \multicolumn{2}{|c|}{0,37} & \multicolumn{2}{|c|}{0,41} & 167,89 & & \multicolumn{2}{|c|}{9,47} \\
\hline & 0,0228 & 9 & 0,0162 & 9 & 0,0169 & 9 & 22,7155 & 9 & 0,9972 & 9 \\
\hline \multirow{2}{*}{ Fícus sp. } & 1,13 & & \multicolumn{2}{|c|}{0,42} & \multicolumn{2}{|c|}{0,45} & 168,12 & & \multicolumn{2}{|c|}{6,70} \\
\hline & 0,0252 & 12 & 0,0471 & 12 & 0,0497 & 12 & 26,4453 & 12 & 1,1156 & 12 \\
\hline \multirow{2}{*}{ Mangifera indica } & 1,16 & & \multicolumn{2}{|c|}{0,52} & \multicolumn{2}{|c|}{0,56} & 126,20 & & 7,4 & \\
\hline & 0,0151 & 9 & 0,0526 & 9 & 0,0596 & 9 & 24,1168 & 9 & 0,9248 & 9 \\
\hline & 1,24 & & 0 & & 0,8 & & 69,86 & & 14 , & \\
\hline C & 0,0164 & 12 & 0,0317 & 12 & 0,0443 & 12 & 7,0698 & 12 & 1,3569 & 12 \\
\hline & 1,18 & & 0 & & 0,6 & & 112,81 & & 15 , & \\
\hline & 0,0257 & 9 & 0,0326 & 9 & 0,0460 & 9 & 10,2770 & 9 & 1,7104 & 9 \\
\hline & 1,17 & & 0 & & 0,6 & & 108,51 & & 13 , & \\
\hline$L t$ & 0,0241 & 9 & 0,0204 & 9 & 0,0360 & 9 & 9,2342 & 9 & 2,7906 & 9 \\
\hline & 1,15 & & 0 & & 0,5 & & 109,22 & & 11 , & \\
\hline$P a c$ & 0,0204 & 15 & 0,0467 & 15 & 0,0593 & 15 & 17,3223 & 15 & 1,8099 & 15 \\
\hline & 1,08 & & 0 & & 0,4 & & 202,15 & & & \\
\hline Chorlsia spec & 0,0438 & 6 & 0,0368 & 6 & 0,0421 & 6 & 28,9277 & 6 & 1,2306 & 6 \\
\hline & 1,07 & & 0 , & & 0,4 & & 203,28 & & & \\
\hline & 0,0266 & 9 & 0,0373 & 9 & 0,0492 & 9 & 35,6553 & 9 & 5080 & 9 \\
\hline
\end{tabular}

Em que: em cada célula ao centro estão os valores médios de cada variável, à esquerda os desvios-padrão e à direita os números de amostras. 
Conforme Melo, Coradin e Mendes (1990), cinco espécies apresentaram massa específica básica abaixo de $0,5 \mathrm{~g} / \mathrm{cm}^{3}$ : Persea americana $\left(0,44 \mathrm{~g} / \mathrm{cm}^{3}\right)$, Spathodea campanulata $\left(0,37 \mathrm{~g} / \mathrm{cm}^{3}\right)$, Ficus $s p$. $\left(0,42 \mathrm{~g} / \mathrm{cm}^{3}\right)$, Chorisia speciosa $\left(0,36 \mathrm{~g} / \mathrm{cm}^{3}\right)$ e Ochoma pyramidale $\left(0,35 \mathrm{~g} / \mathrm{cm}^{3}\right)$, caracterizando-se como madeira leve. Caesalpinea leiostachya, com $0,73 \mathrm{~g} / \mathrm{cm}^{3}$, foi a única com massa específica básica alta, caracterizando-se como madeira dura (Massa específica básica acima de $0,73 \mathrm{~g} / \mathrm{cm}^{3}$ ) e Pterogyne nitens $\left(0,59 \mathrm{~g} / \mathrm{cm}^{3}\right)$, Peltophorum dubium $\left(0,61 \mathrm{~g} / \mathrm{cm}^{3}\right)$, Mangifera indica $\left(0,52 \mathrm{~g} / \mathrm{cm}^{3}\right)$, Syzygium jambolana $\left(0,55 \mathrm{~g} / \mathrm{cm}^{3}\right)$, Ligustrum lucidum $\left(0,55 \mathrm{~g} / \mathrm{cm}^{3}\right)$ e Paquira aquatica $\left(0,50 \mathrm{~g} / \mathrm{cm}^{3}\right)$, com valores entre 0,5 e $0,72 \mathrm{~g} / \mathrm{cm}^{3}$ estão no grupo das madeiras com massa específica média.

A madeira de poda da espécie C. leiostachya $\left(0,73 \mathrm{~g} / \mathrm{cm}^{3}\right)$ pertence à mesma faixa de massa específica da madeiras de Sclerolobium paniculatum $\left(0,7 \mathrm{~g} / \mathrm{cm}^{3}\right)$, que é excelente madeira para a produção de energia e Dalbergia miscolobium $\left(0,72 \mathrm{~g} / \mathrm{cm}^{3}\right)$, ambas encontradas no cerrado (Vale et al., 1996). Para o grupo das espécies com madeira moderadamente dura, Vale et al. (2001) classificaram, entre outras, o Blepharocalix salicifolia com $0,51 \mathrm{~g} / \mathrm{cm}^{3}$ e Stryphnodendron adstringens com $0,55 \mathrm{~g} / \mathrm{cm}^{3}$. Paula e Alves (1997) encontraram, para Peltophorum dubium, massa específica básica de $0,62 \mathrm{~g} / \mathrm{cm}^{3}$ e a classificou entre madeiras de boa qualidade para produção de papel.

Dentre as espécies estudadas, aquelas que apresentaram massa específica de média a alta e podem ser utilizadas para estes fins são: C. leiostachya $\left(0,73 \mathrm{~g} / \mathrm{cm}^{3}\right), P$. nitens $\left(0,59 \mathrm{~g} / \mathrm{cm}^{3}\right), P$. dubium $\left(0,61 \mathrm{~g} / \mathrm{cm}^{3}\right), M$. indica $\left(0,52 \mathrm{~g} / \mathrm{cm}^{3}\right), S$. jambolana $\left(0,55 \mathrm{~g} / \mathrm{cm}^{3}\right)$ e L.lucidum $\left(0,55 \mathrm{~g} / \mathrm{cm}^{3}\right)$. Por outro lado quanto maior a massa específica mais difícil a trabalhabilidade tanto com ferramentas quanto com lixas, restringindo assim o seu uso para produtos que exijam esses tipos de operações, como os pequenos objetos de madeira, que fabricados com base em madeiras de média a baixa massa específica, apresentam facilidade de trabalhabilidade e leveza.

Os valores mais altos em teor de umidade máxima, em base seca, foram observados em C. speciosa e O. pyramidale, em torno de $200 \%$, e os valores mais baixos foram encontrados em C. leiostachya, $P$. nitens e P. dubium.

As espécies: C. leiostachya, S. jambolana, L. lucidum e $P$. dubium apresentaram os maiores valores para contração volumétrica, sendo eles, respectivamente, 14,$45 ; 15,34 ; 13,96$ e $13,26 \%$. Os valores mais baixos foram encontrados em Ficus sp. e M. indica com, respectivamente, 6,70 e 7,41\%.

A massa específica da madeira dos galhos independe do seu diâmetro, uma vez que não foi encontrada diferença significativa ao nível de 5\% de probabilidade para essa variável entre os tratamentos galho fino, médio e grosso.

\section{Análise imediata da madeira}

A Tabela 3 apresenta os valores médios de carbono fixo, materiais voláteis e cinzas de madeira de galho de espécies da arborização de Brasília.

Os teores de material volátil e carbono fixo estão de acordo com a faixa preconizada por Brito \& Barrichelo (1982), de 75 a $85 \%$ para o primeiro e de 15 a $25 \%$ para o segundo, à exceção de Persea americana e Ochroma piramidales. Observa-se, pelos resultados, que, em geral, as madeiras de maiores massas específicas apresentaram maiores teores de carbono fixo. Madeiras com maiores teores de carbono fixo têm uma queima mais lenta que aquelas com elevados teores de materiais voláteis. Os maiores teores de carbono fixo foram verificados em P. nitens $(18,45 \%), P$. dubium $(17,43 \%)$ e C. leiostachya $(16,77 \%)$, madeiras com altas massas específicas. O mais baixo teor de carbono fixo foi encontrado em $O$. pyramidale $(12,56 \%)$ apresentando menor massa específica e, por outro lado, mostrou ter o maior valor de materiais voláteis $(86,69 \%)$.

Os maiores valores para teor de cinzas variaram entre $1,5 \%$ (C. speciosa) e 0,20\% (C. leiostachya), os quais se enquadram dentro da faixa comumente encontrada para folhosas.

A massa específica e o teor de carbono fixo podem ser utilizados como indicadores de madeira para combustão direta, conforme Brito e Barrichelo (1978), citando Junge (1975), Arola (1976) e Corder (1976). As espécies C. leiostachya, P. nitens, P. dubium, M. indica, S. jambolana e L. lucidum apresentam, em geral, elevada massa específica e elevado teor de carbono fixo. 
TABELA 3: Valores médios de material volátil (MV), teor de cinzas (CZ) e teor de carbono fixo (CF) para madeiras de galhos de espécies utilizadas na arborização de Brasília, DF.

TABLE 3: Average values of volatile material content (MV), ash content (CZ) and fixed carbon content (CF) from Brasília wood branches.

\begin{tabular}{l|c|c|c}
\hline \multicolumn{1}{c|}{ Espécie } & MV (\%) & CZ (\%) & CF (\%) \\
\hline Persea americana & 85,61 & 0,35 & 14,04 \\
Pterogyne nitens & 81,00 & 0,55 & 18,45 \\
Peltophorum dubium & 81,20 & 1,37 & 17,43 \\
Spathodea campanulata & 82,11 & 1,16 & 16,74 \\
Ficus sp. & 82,83 & 0,93 & 16,24 \\
Mangifera indica & 83,74 & 0,87 & 15,39 \\
Caesalpinea leiostachya & 81,81 & 1,43 & 16,77 \\
Syzygium jambolana & 83,57 & 0,20 & 16,23 \\
Ligustrum lucidum & 83,13 & 1,10 & 15,77 \\
Paquira aquática & 83,87 & 1,06 & 15,08 \\
Chorisia speciosa & 82,92 & 1,50 & 15,58 \\
Ochoma pyramidale & 86,85 & 0,59 & 12,56 \\
\hline
\end{tabular}

\section{Carbonização da madeira}

A Tabela 3 apresenta os valores médios para os rendimentos da carbonização das espécies de árvores da arborização de Brasília.

As espécies que mais produziram carvão vegetal foram $S$. campanulata $(32,22 \%)$ e C. speciosa $(31,85 \%)$, e as que menos produziram foram $L$. lucidum $(27,41 \%)$ e $P$. aquatica $(27,84 \%)$. O maior valor para rendimento de licor pirolenhoso foi observado em $L$. lucidum $(55,84 \%)$, sendo que as espécies $S$. campanulata $(27,81 \%)$ e $P$. aquatica $(27,84 \%)$ foram as que mais produziram gases não condensáveis.

Os valores para rendimento gravimétrico estão dentro do que foi encontrado por Pastore et al. (1989), usando metodologia semelhante, em que a variação ficou em torno de 27 a 37\%, trabalhando com vinte espécies da Amazônia.

Para rendimento de licor pirolenhoso, as espécies ficaram com valores acima de $45 \%$, com exceção de $S$. campanulata, com 39,97. A espécie, que apresentou maior valor foi $L$. lucidum com 55,85\%, acima do rendimento encontrado por Vale et al. (1996) para jacarandá do cerrado (Dalbergia miscolobium), que foi de $54,31 \%$, com a mesma metodologia.

S. campanulata apresentou o maior valor para rendimento de gases não-condensáveis, $27,81 \%$, e os valores mais baixos foram $15,98 \%$ para $S$. campanulata e $16 \%$ para C. leiostachya, S. jambolana, L. lucidum e O. pyramidale, valores esses comparáveis à Sclerolobium paniculatum, com $16,15 \%$, segundo Vale et al. (1996).

Via de regra, todas as espécies podem ser utilizadas para a produção de carvão, no entanto, aquelas que apresentam maiores massas específicas e maiores rendimento gravimétrico devem ser preferidas. 
TABELA 4: Valores médios dos rendimentos da carbonização.

TABLE 4: Average values of carbonisation.

\begin{tabular}{|c|c|c|c|c|c|c|}
\hline \multirow{3}{*}{$\begin{array}{l}\text { Amostra } \\
\text { Persea americana }\end{array}$} & \multirow{2}{*}{\multicolumn{2}{|c|}{$\frac{\mathrm{RG}(\%)}{26.04}$}} & \multirow{2}{*}{\multicolumn{2}{|c|}{$\frac{\text { RLP (\%) }}{5376}$}} & \multicolumn{2}{|c|}{ RGNC (\%) } \\
\hline & & & & & 20, & \\
\hline & 3,1092 & 3 & 8,2951 & 3 & 6,0913 & 3 \\
\hline \multirow[b]{2}{*}{ Pterogyne nitens } & \multicolumn{2}{|c|}{29,91} & \multicolumn{2}{|c|}{54,11} & \multicolumn{2}{|c|}{15,98} \\
\hline & 0,7148 & 3 & 2,0454 & 3 & 12,1397 & 3 \\
\hline \multirow{2}{*}{ Peltophorum dubium } & \multicolumn{2}{|c|}{29,51} & \multicolumn{2}{|c|}{48,06} & \multicolumn{2}{|c|}{22,44} \\
\hline & 1,5994 & 2 & 15,1280 & 2 & 26,7253 & 2 \\
\hline \multirow{2}{*}{ Spathodea campanulata } & \multicolumn{2}{|c|}{32,22} & \multicolumn{2}{|c|}{39,97} & \multicolumn{2}{|c|}{27,81} \\
\hline & 1,3081 & 3 & 9,8486 & 3 & 8,4459 & 3 \\
\hline \multirow{2}{*}{ Ficus sp. } & \multicolumn{2}{|c|}{28,06} & \multicolumn{2}{|c|}{50,87} & \multicolumn{2}{|c|}{21,06} \\
\hline & 0,8530 & 3 & 4,7462 & 3 & 5,0207 & 3 \\
\hline \multirow{2}{*}{ Mangifera indica } & \multicolumn{2}{|c|}{29,66} & \multicolumn{2}{|c|}{54,87} & \multicolumn{2}{|c|}{15,47} \\
\hline & 0,9402 & 3 & 2,4801 & 3 & 2,4938 & 3 \\
\hline \multirow{2}{*}{ Caesalpinea leiostachya } & \multicolumn{2}{|c|}{29,59} & \multicolumn{2}{|c|}{52,70} & \multicolumn{2}{|c|}{17,70} \\
\hline & 2,1157 & 3 & 0,6429 & 3 & 1,9700 & 3 \\
\hline \multirow{2}{*}{ Syzygium jambolana } & \multicolumn{2}{|c|}{29,94} & \multicolumn{2}{|c|}{53,11} & \multicolumn{2}{|c|}{16,94} \\
\hline & 1,7066 & 3 & 2,6874 & 3 & 3,8759 & 3 \\
\hline \multirow{2}{*}{ Ligustrum lucidum } & & & & & 16 & \\
\hline & 1,1348 & 3 & 1,6082 & 3 & 0,6424 & 3 \\
\hline & & & & & 26 , & \\
\hline Paquira aquatıca & 0,7795 & 3 & 5,8369 & 3 & 5,1044 & 3 \\
\hline & & & & & 16 & \\
\hline Chorlsia speciosa & 0,8822 & 2 & 1,5001 & 2 & 0,6184 & 2 \\
\hline & & & & & 21 & \\
\hline Ochoma pyramidale & 2,1072 & 3 & 1,4741 & 3 & 3,5103 & 3 \\
\hline
\end{tabular}

Em que: em cada célula ao centro estão os valores médios de cada variável, à esquerda o desvio-padrão e à direita o número de amostras; $\mathrm{RG}=$ rendimento gravimétrico; $\mathrm{RLP}=$ rendimento em licor pirolenhoso; $\mathrm{RGNC}=$ rendimento em gases não-condensáveis na CNTP (condição normal de temperatura e pressão).

\section{Relação da massa específica com outras propriedades}

A Tabela 5 apresenta as equações de regressão linear entre a massa específica básica e algumas características da madeira de galhos, e as Figuras: 1A e 1B; $2 \mathrm{C}$ e $2 \mathrm{D} ; 3 \mathrm{E}$ e $3 \mathrm{~F}$; $4 \mathrm{G}$ e $4 \mathrm{H}$ mostram o comportamento entre tais variáveis.

TABELA 5: Equações de regressões para características da madeira, da carbonização e da qualidade do carvão.

TABLE 5: Regression equations for wood characteristics, carbonisation yield and charcoal quality.

\begin{tabular}{l|c|c}
\hline Equação & Coeficiente de determinação & Significância \\
\hline $\mathrm{Cv}=4,08655+13,81781 \mathrm{Meb}$ & $\mathrm{R}^{2}=0,3298$ & $*$ \\
Umáx $=316,91002+366,75294 \mathrm{Meb}$ & $\mathrm{R}^{2}=0,9043$ & $*$ \\
$\mathrm{RG}=30,05176-1,86578 \mathrm{Meb}$ & $\mathrm{R}^{2}=0,0099$ & $\mathrm{~ns}$ \\
$\mathrm{RLP}=41,73003+18,14181 \mathrm{Meb}$ & $\mathrm{R}^{2}=0,1210$ & $*$ \\
$\mathrm{RGNC}=28,224-16,28685 \mathrm{Meb}$ & $\mathrm{R}^{2}=0,0958$ & $*$ \\
$\mathrm{Cf}=12,15906+7,35842 \mathrm{Meb}$ & $\mathrm{R}^{2}=0,3037$ & $*$ \\
$\mathrm{Mv}=87,14862-7,81815 \mathrm{Meb}$ & $\mathrm{R}^{2}=0,2788$ & $*$ \\
$\mathrm{Cz}=0,69131+0,46671 \mathrm{Meb}$ & $\mathrm{R}^{2}=0,0159$ & $*$ \\
\hline
\end{tabular}

Em que: Meb = Massa Específica; RG = Rendimento Gravimétrico; RLP = Rendimento em Licor Pirolenhoso; RGNC

$=$ Rendimento em Gases Não-Condensáveis; $\mathrm{Cf}=$ Carbono Fixo; $\mathrm{Mv}=$ Material Volátil; $\mathrm{Cz}=$ Teor de Cinzas; $\mathrm{CV}=$ contração volumétrica; $*$ = significativo à $5 \%$ de probabilidade; Umáx $=$ Unidade máxima, ns = não significativo. 


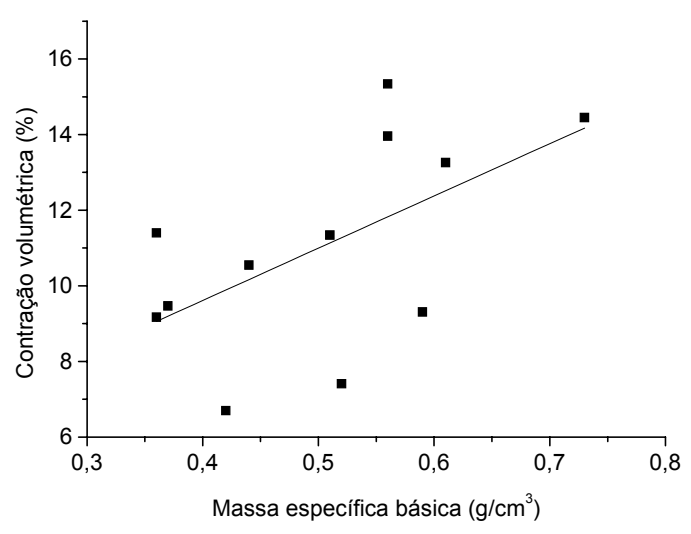

A

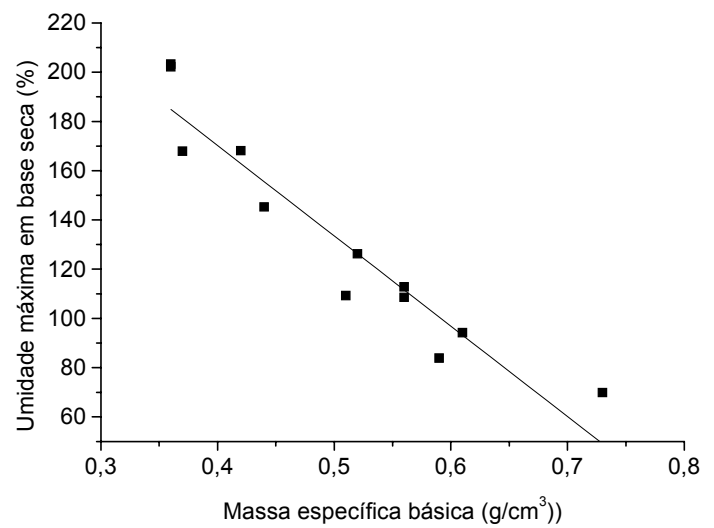

B

FIGURA 1: Contração (A) e umidade máxima (B) em função da massa específica da madeira.

FIGURE 1: Contraction (A) and wood moisture content (B) in relation to basic density.

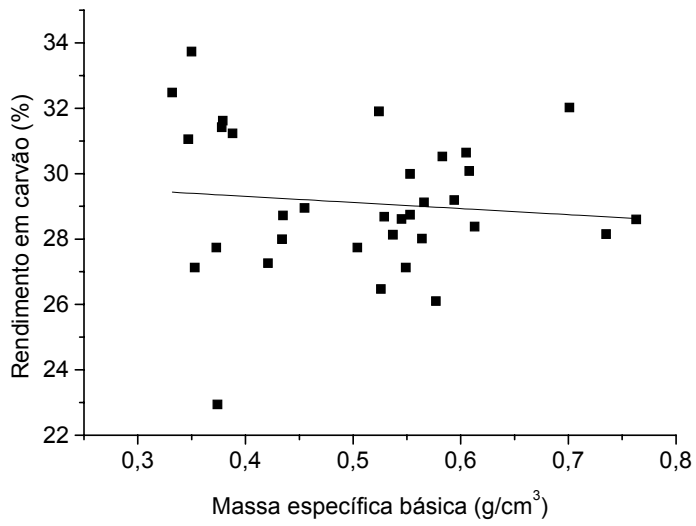

$\mathrm{C}$

FIGURA 2: Rendimento em carvão (C) e licor pirolenhoso (D) em função da massa específica básica. FIGURE 2: Charcoal yield (C) and pyrolig neous liqueur (D) in relation to basic density.

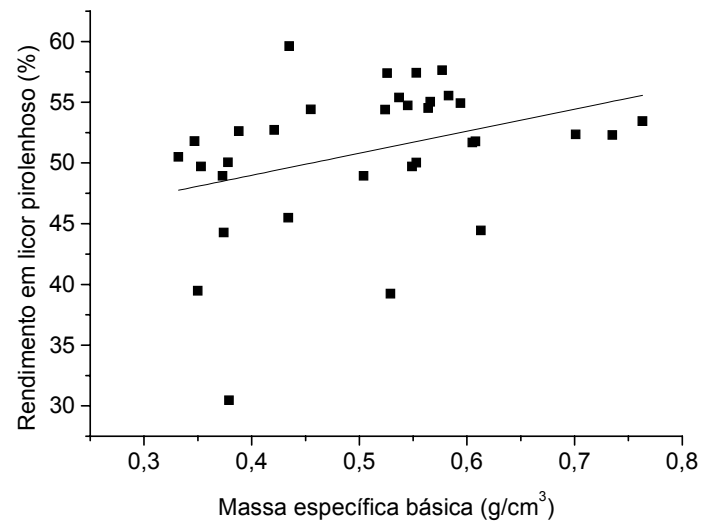

D

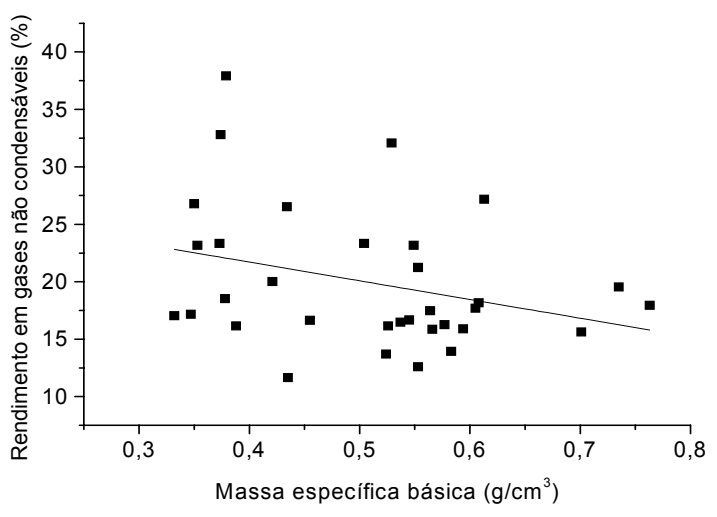

E 


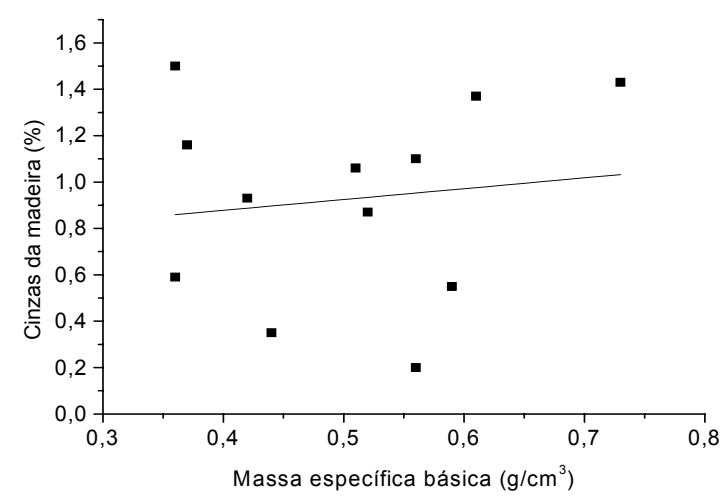

G

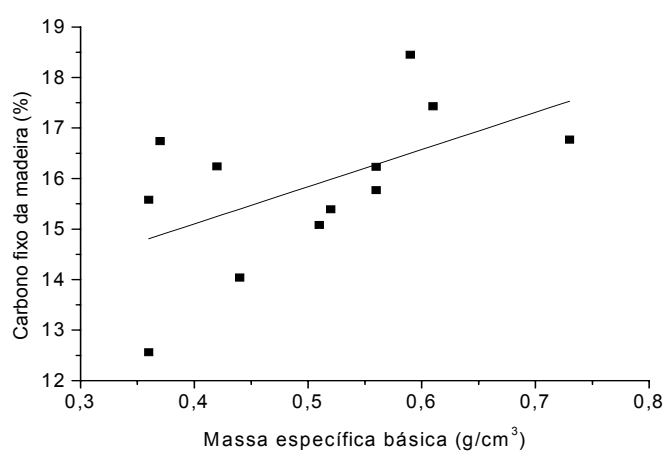

$\mathrm{H}$

FIGURA 4: Teor de cinzas $(\mathrm{G})$ e do teor de carbono fixo $(\mathrm{H})$ em função da massa específica básica. FIGURE 4: Ash $(\mathrm{G})$ and fixed carbon $(\mathrm{H})$ in relation to basic density.

Como se esperava, a massa específica tem uma relação direta com a contração volumétrica da madeira e inversa com o teor de umidade. Não há uma relação significativa entre rendimento em carvão vegetal e a massa específica básica da madeira. Comportamento semelhante foi encontrado por Brito e Barrichelo (1977), quando trabalharam com espécies de Eucalyptus. A relação entre a massa específica básica da madeira e o rendimento em licor pirolenhoso e o rendimento em gases não-condensáveis, é fraca, com baixos valores para coeficiente de determinação. Observa-se que 30\% dos valores encontrados para carbono fixo e $27 \%$ dos valores encontrados para materiais voláteis são explicados pela variação da massa específica básica da madeira a um nível de significância de 5\%.

\section{CONCLUSÃO}

As espécies estudadas, com exceção de Caesalpinea leiostachya, possuem madeiras medianamente macia.

Considerando que o melhor uso da madeira para produção de energia na forma de calor está ligada a maiores massas específicas, maiores teores de carbono fixo e menores teores de materiais voláteis, destacamse as seguintes espécies: Caesalpinea leiostachya; Pterogyne nitens, Peltophorum dubium, Mangifera indica, Syzygium jambolana e Ligustrum lucidum que apresentam características favoráveis tanto para o uso como lenha, quanto para a produção de carvão vegetal.

Não foi encontrada relação de causa e efeito entre a massa específica básica e a produção de carvão vegetal, mas, por outro lado, se encontrou relação positiva entre essa variável e o teor de carbono fixo.

Os baixos valores dos coeficientes de determinação para os rendimentos da carbonização, incluindo a não significância para o rendimento gravimétrico, indicam que outros fatores além da massa específica básica da madeira têm influência nesses rendimentos.

\section{REFERÊNCIAS BIBLIOGRÁFICAS}

ASSOCIAÇÃO BRASILEIRA DE NORMAS TÉCNICAS. MB-26/1940 : ensaios físicos e mecânicos de madeiras. Rio de Janeiro: 1940. 15p.

ASTM - American National Standard. ASTM D 1762 - 64 (Reapproved 1977) : chemical analysis of wood charcoal. 1977. p. $577-579$.

BRITO, J.O ; BARRICHELO, L.E. Correlações entre características físicas e químicas da madeira e a produção de carvão vegetal: I. Massa específica e teor de lignina da madeira de eucalipto. IPEF, n. 14, p. 47-56, 1977.

BRITO, J.O.; BARRICHELO, L.E. Características do eucalipto como combustível: análise química e imediata da madeira e da casca. IPEF, v. 16, p. 63-78, 1978.

BRITO, J.O.; BARRICHELO, L.E. Aspectos técnicos da utilização da madeira e carvão vegetal como combustíveis. In : SEMINÁRIO DE ABASTECEMENTO ENERGÉTICO INDUSTRIAL COM RECURSOS FLORESTAIS, 2,1982, São Paulo. Anais ...São Paulo, 1982. p. 101-137. 
FOELKEL, C.E.B; BRASIL, M.A.M.; BARRICHELLO, L.E.G. Métodos para determinação da massa específica básica de cavacos para conífera e folhosas. IPEF, n. 2/3, p. 65-74, 1971.

JACINTO, J. M. M. Análise silvicultural urbana de seis espécies florestais utilizadas na arborização de Brasília. 2001. 55p. Dissertação (Mestrado em Engenharia Florestal) - Universidade de Brasília,Brasília, 2001.

MELO, J.E.; CORADIN, V.R. ; MENDES, J.C. Classes de densidade para madeiras da Amazônia brasileira. VI In : CONGRESSO FLORESTAL BRASILEIRO, 6., 1990, Campos do Jordão. Anais...Campos do Jordão, 1990. v. 3, p. 695-705.

PASTORE, T.C.M.; OKINO, E.Y.A.; PASTORE JUNIOR, F.P. Carbonização de madeiras da Amazônia. Parte I: Floresta Nacional do Tapajós. Brasília: IBAMA, Laboratório de Produtos Florestais, 1989. 12p. (Série Técnica, 12)

PAULA, J.E.; ALVES, J.L.H. Madeiras nativas : anatomia, dendrologia, dendrometria, produção e uso. 1997.

VALE, A.T.; COSTA, A.F.; GONÇALEZ, J.C.; NOGUEIRA, M.V.P. Relações entre a massa específica básica da madeira, o rendimento e a qualidade do carvão vegetal de espécies do cerrado. Revista Árvore, v. 25, n. 89, p. 89-95, 2001 .

VALE, A.T.; NOGUEIRA, M.V.P.; SILVA, M.A. Rendimento da carbonização e qualidade do carvão vegetal de madeiras do cerrado em comparação ao Eucalyptus grandis. Revista Árvore, v. 20, n. 1, p. 93-99, 1996.

VITAL, B.R.. Métodos para determinação do teor e umidade da madeira. Boletim Técnico SIF, Viçosa, n. 13, p. 1-33, 1997.

VITAL, B.R. Método de determinação da massa específica da madeira. Boletim Técnico SIF, Viçosa, n. 2, p. 1- 21, 1984. 